\title{
A new interlink decision making index for making multi-criteria decisions
}

Eric Hu (School of Engineering and Technology, Deakin University, Australia), Linda Zou(School of Engineering and Technology, Deakin University, Australia) and Craig Langston (Professor, School of Architecture and Building, Deakin University, Australia).

\section{ABSTRACT}

Multi-criteria decisions usually require measurement or evaluation of performance in different units and their mix by application of weighting factors. This approach leads to potential manipulation of the results as a direct consequence of the applied weightings. In this paper a mechanism that is the brain child of the authors, has been proposed to overcome this problem. It is known as the Interlink Decision Making Index (IDMI) and has all the desired features: simple, interlink (all criteria) and automatically guaranteed dominant influence of critical criteria (i.e. no human weighting needed). The IDMI is capable of reflecting the total merits of a particular option once the normal decision making criteria and (up to two) critical criteria have been chosen. Then, without arbitrarily weighting criteria, comparison and selection of the best possible option can be made. Simple software has been developed to do this numerical transfer and graphic presentation. Two hypothetical examples are presented in the paper to demonstrate the application of the IDMI concept and its advantages over the traditional "tabular and weighting method" in the decision making process.

Keywords: multi-criteria decision making, critical criteria, sustainability, interlink decision making index, visualizing

\section{INTRODUCTION}

There are many assessment techniques that generate outcomes to feed into the decision making process. For example, assessments focusing on development project proposals (e.g. environment impact assessments) and single processes or production lines (e.g. waste minimization assessments) are designed to assist in the selection of location specific environmental policy. The assessment of pollution (e.g. risk assessments) will assist with specific regulation compliance. Life cycle assessment (LCA) assists new products or production processes to be evaluated from an environmental point of view (Weidema, 2000). In addition, direct or indirect economic assessment models will feed full economic interpretation. All these assessments provide large reports and may recommend different options in particular cases, and as a consequence simple economic criteria often dominate or the decision is made arbitrarily based on subjective judgment or intuition. Several methodologies and algorithms have been developed to provide decision makers with advice about selection, but they are either complicated or expensive to use (Vignes, 1999; Khan F.I. et al., 2002), or narrowly focused (Chen and Huang, 2001). Moreover, in the traditional decision making process, "weighting" each criterion is a very difficult process and depends heavily on the personal preference of the decision maker, despite arguments that the weighting process is fundamental to traditional multiple criteria decision making (MCDM). There is a need to provide decision makers with a simple, less human-interfered (yet still transparent) mechanism that integrates all the key criteria (environmental, economic, technical, safety etc.) while allowing a couple of them to be chosen as critical criteria (CC) that are more influential than others in the decision making process. The proposed solution, the Interlink Decision Making Index has all these features: simple, interlink (all criteria) and automatically guaranteed dominant influence of CC (i.e. no human weighting needed).

\section{Multi-Criteria Analysis}

In normal life people are faced with decisions on a regular basis. The process of decision making involves identifying, comparing and ranking options using multiple criteria. Often this process occurs without even thinking much about it. However, for large investment-related problems there is a tendency to simplify the decision process into a single monetary criterion. The selected option is the one that demonstrates the highest monetary value.

Project appraisal techniques are employed to structure the diverse array of data into a manageable form and provide an objective and consistent basis of choosing the best solution for a situation. Cost-benefit analysis (CBA) is a respected appraisal technique that is widely used by both public and private organizations to aid in complex investment decisions. In conventional CBA much effort has been made to assess the input costs and output benefits by means of a market approach. With the increasing awareness of possible negative external effects and the importance of distributional issues in economic development, the usefulness of CBA has become increasingly controversial. In response to this dissatisfaction, recent attention has been paid to multi-dimensional evaluation approaches (Nijkamp et al., 1990).

The identification of value for money on development projects is still related to monetary return. But other issues are also relevant, particularly for social infrastructure projects, and some are becoming increasingly significant. For example, issues such as functionality and resource efficiency are vital to the assessment of sustainable development in the wider social context. Since no single criterion can adequately address all the issues involved in complex decisions of this type, a multicriteria approach to decision making offers considerable advantage.

Social costs and benefits (including those related to environment impact) need to be integrated into the evaluation process and a strategy developed that will give these factors proper consideration in practice. Social costs and benefits should not be discounted alongside conventional cash flows as they bear little relationship to financial matters and do not 
reduce in importance exponentially over time. In fact, future generations may value environmental issues more highly than the present generation.

A multi-criteria approach is better suited to deal with the complexities of sustainability assessment. Key criteria such as investment return, functional performance, energy usage and loss of habitat can be combined to form a single decision model that can rank options according to the perceived level of achievement of sustainable development objectives (Ding and Langston, 2002). Such an approach moves away from conventional economic evaluation and the inherent problems associated with monetary assessment.

Traditional cost-benefit analysis uses a single critical criterion as the main tool to evaluate projects based on market transaction. However, over the past decade criticism has stemmed from attempts at putting underlying welfare economic theory into practice. It is often difficult or even impossible to improve social welfare in a society if the natural environment continues to be consumed and depleted. The CBA framework ignores and/or under-estimates environmental assets as there are considerable difficulties in measuring all relevant impacts of a project in monetary units (Abelson, 1996).

Intangible and externalities have become major components of concern in project development decisions, in particular due to the possibility of undesirable effects on the natural environment. The presence of externalities, risks and spillovers generated by project development preclude a meaningful and adequate use of a market approach methodology. When the analysis turns to such effects as environmental quality and loss of biodiversity, it is rarely possible to find monetary variables that can provide a valid indicator. Although effort has been made to arrive at values for social costs and benefits, in practice it is almost impossible to place anything more sophisticated than arbitrary numerical values on such effects. The requirement for incorporating environmental issues into project appraisal process is increasing, and so the imputation of market prices becomes more and more questionable.

Alternatives have been developed to replace CBA completely with other techniques that do not require social costs and benefits to be monetarized. Cost effectiveness analysis (CEA) and environmental impact assessment (EIA) are leading solutions in this respect. Others have suggested supplementing CBA with a technique that can measure environmental costs in different ways (Nijkamp et al., 1990; Abelson, 1996; Van Pelt, 1993; Hanley, 1992). Multiple criteria decision making (MCDM) is widely accepted to aid the evaluation process in this respect.

MCDM is thus a technique designed to value two or more criteria and it is particularly useful for those social impacts that cannot be easily quantified in normal market transactions. MCDM transfers the focus of measuring criteria with prices to applying weights and scores to these impacts to determine a preferred outcome. The total scores that reflect the importance of environmental impacts are used to rank project options. MCDM is a more flexible methodology and can deal with quantitative, qualitative and mixed data, whilst CBA may only handle data of a quantitative nature. In contrast to CBA, MCDM does not impose any limitation in the number and nature of criteria. Therefore, MCDM is a more realistic methodology in dealing with the growing complexity of development decisions. However MCDM arguably suffers from one noticeable problem - the determination of weighting factors is dependent on the judgment of the decision maker and has the potential to lead to bias and political manipulation. The approach described later in this paper aims to overcome this deficiency.

\section{INTERLINK DECISION MAKING INDEX (IDMI)}

The proposed IDMI is a numerical value that has no unit or physical meaning. It is used only to compare the options of a particular case (i.e. new product, manufacturing process or a construction project) under similar selection criteria. The smaller the value of IDMI (for a particular option), the better the option may be. The IDMI value is always accompanied by an illustration to show the magnitude of each criterion.

In principle, the IDMI can be understood as something similar to the area or volume (or other geometrical properties) of a triangle (2D) or a tetrahedron (3D) that is constructed by all the criteria in such a way that the critical criteria (CC) are more influential and more decisive to the IDMI value while still incorporating other identified criteria. The proposed methodology supports up to two critical criteria, but where more criteria are considered important it is recommended to treat all criteria equally.

In the calculation of IDMI, there is an option to choose none, one or two critical criteria out of the selection criteria. If no CC is chosen, the calculation of IDMI will then have all selection criteria treated the same. If one or two CC is chosen, then they will be assigned more weight in the IDMI calculation.

Mathematically, if there are $n$ selection criteria in a decision making case, the IDMI values are calculated as:

With no CC:

$$
I D M I=\sum_{i=1}^{n} C i
$$

With one CC:

$$
I D M I=\frac{1}{2}\left(\sum_{i=1}^{n-1} C i\right) C_{n}
$$

with two CCs:

$$
I D M I=\frac{1}{3}\left(\sum_{i=1}^{n-2} C_{i}\right) C_{n-1} C_{n}
$$

where:

$\mathrm{C}_{\mathrm{n}}=$ (the value) of the first $\mathrm{CC}$

$\mathrm{C}_{\mathrm{n}-1}^{\mathrm{n}}=$ the second $\mathrm{CC}$;

$n=$ the number of selection criteria. 


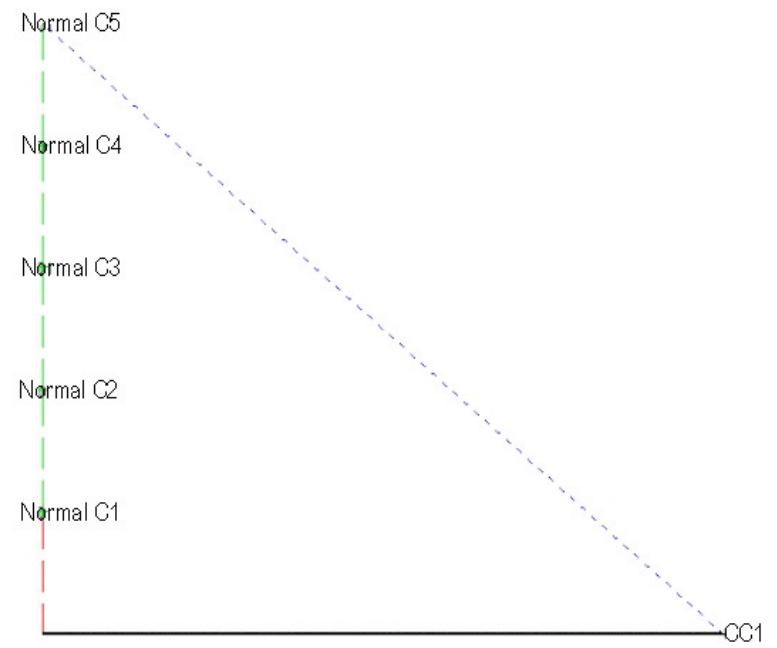

Figure 1a: Area equals the IDMI with $1 \mathrm{CC}$, in a total 6 criteria case

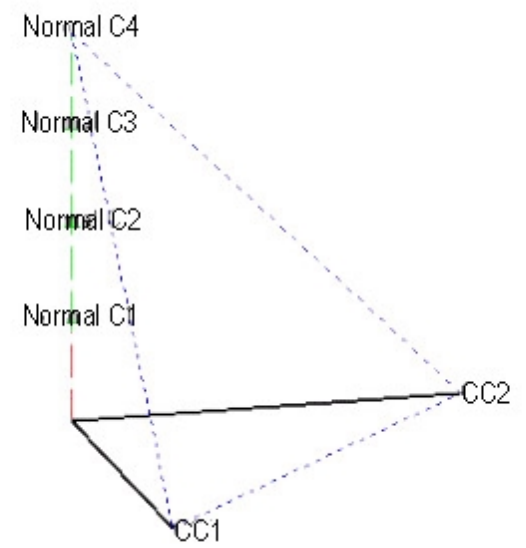

Figure $1 b$ : Volume equals the IDMI with $2 \mathrm{CCs}$, in a total 6 criteria case

The logic behind the formula is that for $1 \mathrm{CC}$ case, the IDMI is actually the area of a right-angled triangle made by the sum of $(\mathrm{n}-1)$ normal criteria as one side and the $\mathrm{CC}$ as the another side of the triangle, as shown in Fig. 1a. In this way, all criteria contribute to the "area" ie. IDMI value, but the CC is critical and contributes more. If two criteria (out of total $n$ ) were more important than others (in the decision making process) and were selected as CC, the IDMI is then the volume of the rightangled triangular pyramid made from $2 \mathrm{CCs}$ and the sum of all normal criteria as shown in the Fig. $1 \mathrm{~b}$.

To make the IDMI concept more transparent, the IDMI values are always accompanied by an illustration to show visually the features of the decision model.

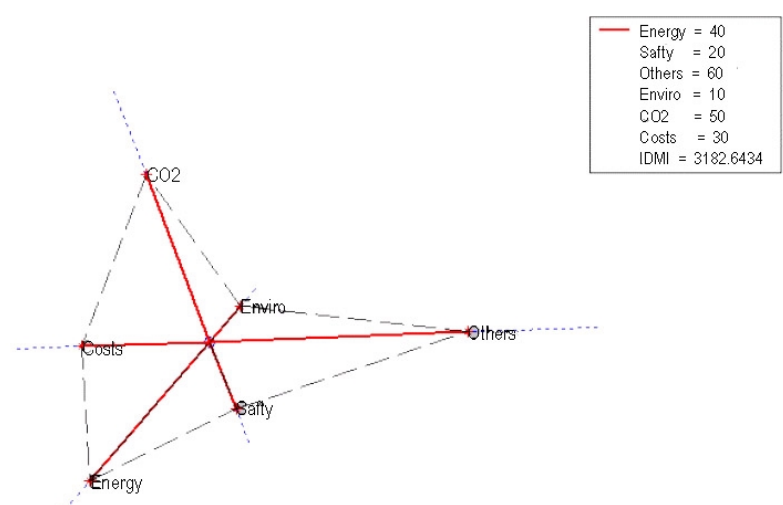

Figure 2.a.: Illustration of IDMI with no CCs 


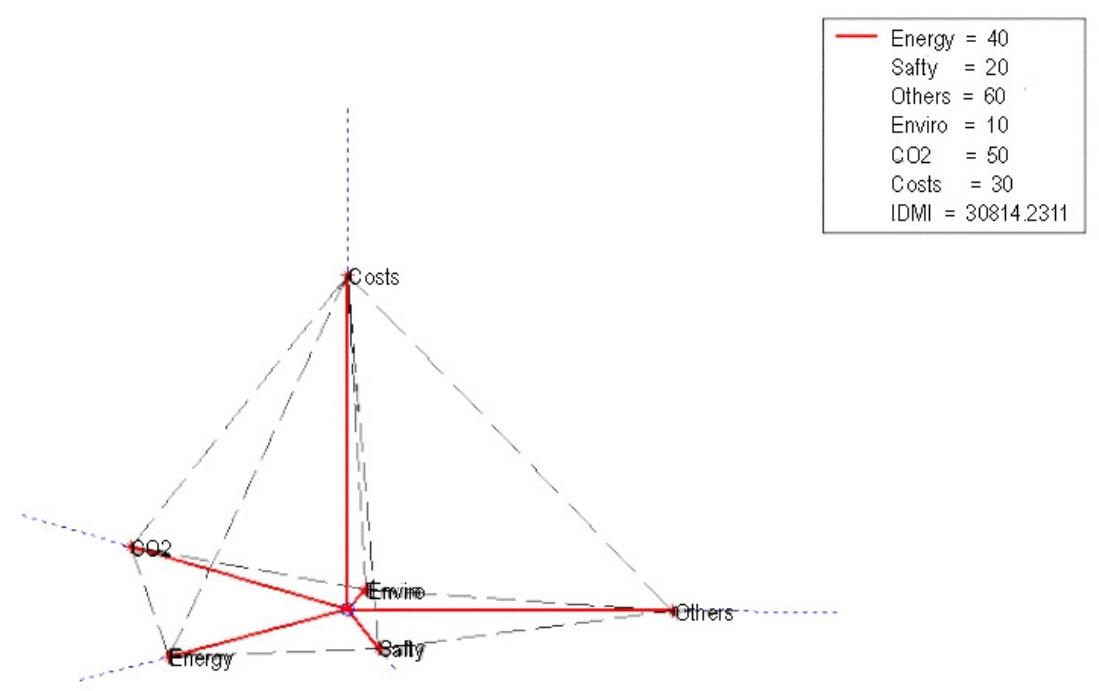

Figure 2 b.: A sample illustration of IDMI with one CC in a total of 6 criteria.

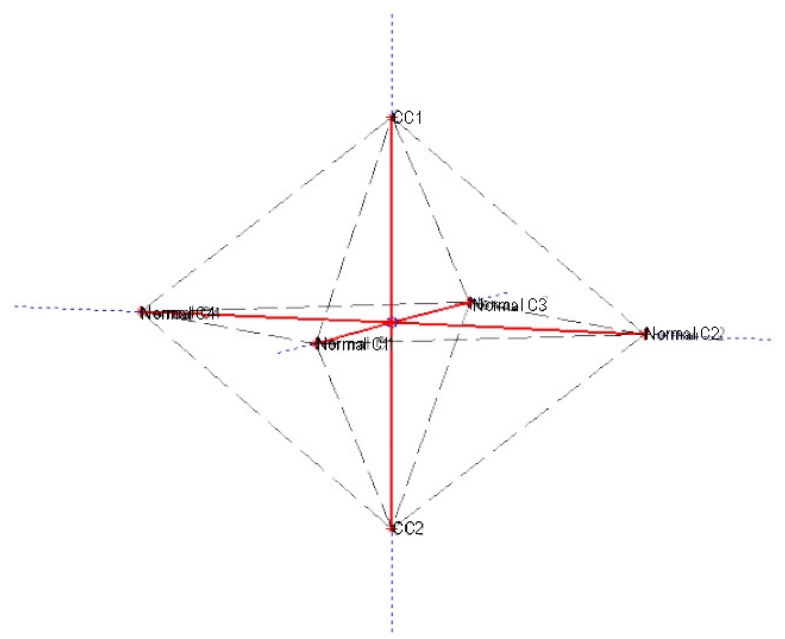

Figure 2c.: A sample illustration of IDMI with two CCs in a total of 6 criteria.

illustrations of IDMI are:

for no CC, the illustration is a "star" diagram with all criteria evenly distributes on a plan, as shown in the Fig.2a;

for one CC, it is a 3-D structure with CC as the height, as shown in Fig. 2b;

for two CCs, the illustration is a "octahedron" made by the normal criteria in plan and two CCs (on opposite directions) sitting at origin at right angles to the plan, as shown in Fig. 2c;

It should be noted that the IDMI values (i.e. equations 1-3) are not the "area" or "volume" of the illustration because they do not mathematically guarantee the more influential position of CC(s) compared to normal criteria. As the principle underlying the IDMI concept, CC should be guaranteed to be more sensitive and contribute more to the IDMI than normal criteria. There are no direct (mathematical) links between the IDMI value and the illustration. The main purpose of the illustration is to visualize the relative desirability of different options.

IDMI makes sense only if comparing two or more options using the same criteria (with the same CCs). It is not valid to calculate the IDMI value for option 1 using two $C C$ and calculate the IDMI value for option 2 using only one $\mathrm{CC}$ and then compare them.

The sensitivity of critical and normal criteria on IDMI will depend on the number of the criteria selected and the actual values of all criteria, but in principle CC is always more sensitive and influential than normal criteria.

At the conceptual level, the proposed IDMI is thought to have the following characteristics:

1) It is simple and effective. It provides the decision makers with a uniform scale to measure the various options.

2) No weighting inputs from the decision maker are needed for $\mathrm{CCs}$, as they are built into the IDMI structure, although the decision maker still needs to determine which criteria should be chosen as $\mathrm{CC}(\mathrm{s})$. Therefore the arbitrary human influence in the weighting process will be minimal.

3) Another feature of the IDMI concept is that the weighting factors are dependent on the number of the criteria (and their values) selected and their scale, i.e. the weighting factors will vary but can still maintain the criticality of the CC. 
4) It provides the flexibility for the decision makers to look into the merits of the options when choosing different criteria and priorities.

5) It provides a single value for decision making and visualizes the criteria in the options. It will also help to understand what is the "trade-off" when a decision is being made. The concept can be used at various levels in the decision making process (e.g. from micro project to macro government decisions

\section{CALCULATION OF IDMI}

\section{Data Generation}

To start to calculate the IDMI, the selection of criteria (and CC) is the first step. This selection can be done by a survey of managers or decision makers in industry or government or from experience of the particular decision making group. This is not a weighting process but an identification process.

IDMI is a post assessment evaluation concept, so it needs data feeding from the various assessments to function. The initial data input to each criterion for each option is very important. The data is normally generated from the various assessments such as LCA, EIA or CBA. It is logical to collect data for all possible options but in practice the selection is more confined.

As the values obtained for each criterion will have different units and magnitudes, it is difficult to compare them directly. It is essential to make the values dimensionless before the IDMI calculation. There are various ways to do this, and these are described below:

1) For each criterion, find the ratio of the data to a reference point at the same category. For example, in the manufacture of new product (options 1 and 2), $\mathrm{A}$ and $\mathrm{B}$ tons of $\mathrm{CO}_{2}$ will be generated per unit of production respectively, but for the same manufacturing process, current world best practice (reference point) emits just $\mathrm{C}$ tons of $\mathrm{CO}_{2}$. So the ratio of $\mathrm{A} /$ $\mathrm{C}$ and $\mathrm{B} / \mathrm{C}$ (without any unit) can be used as the input value of a $\mathrm{CO}_{2}$ criterion to calculate IDMI. It is also possible to use one of the options as the reference case.

2) All data can be converted to cost (or other units in some cases), based on rational assumptions. For example, $\mathrm{CO}_{2}$ emissions may be converted to cost by multiplying by the carbon tax rate set by government. Wastewater discharged may be converted by considering the costs of installing and operating necessary treatment facilities, etc. Safety is a difficult criteria to convert but it might be transferred to cost by considering the insurance premium or health liability.

3) Alternatively input the value of each criterion directly and ignore the units, e.g. for $A$ tons of $\mathrm{CO}_{2}$, directly input $A$, and $\$ B$ of expenditure simply input $B$. As long as the same method of conversion is used to generate the data in all options, the calculated IDMI can reliably compare options and help reach balanced decisions.

For the IDMI calculation, the same formula should be applied to all options. The ultimate objective of IDMI is to provide a score to help the decision maker select among competing options.
IDMI calculation and visualizing software

Computer software to calculate the IDMI defined in this paper has been developed by the authors. The software, based on MatLab, is able to generate the diagram of the IDMI illustration and to calculate the IDMI values from the users' choice of criteria and input data. The software can also generate the results under various scenarios and place various options side by side for comparison. It allows the user to view the different IDMI values between options when different criteria are selected as CCs.

During the decision making process, the minimum IDMI is the preferred option. If two options have similar IDMI values, the decision maker may then examine the details of the IDMI illustration to understand its characteristics. The software can generate the overlapped IDMI illustrations to visually compare the options. Now the authors are in the process to develop the software further to include a comprehensive report interprets the analysis and graphical results for the users.

\section{A HYPOTHETICAL EXAMPLE USING IDMI IN DECISION MAKING}

Assume there are four options for developing a new power plant and the previous assessments had been completed to give each option a score or figure for each criterion. The decision of selecting an option will be based on the following six criteria:

1) Capital cost

2) $\mathrm{CO}_{2}$ emission

3) Plant efficiency

4) Occupational heath and safety $(\mathrm{OH} \& \mathrm{~S})$ risks

5) Sustainability

6) Other losses/risks

Table 1 shows the values (from previous assessments) for each criterion in each option. The values are the ratios to the reference case (i.e. Option 1). Given the principle that a smaller IDMI is better, the criteria data needs to be designed/converted in such a way that the better practice appears as a smaller value. For example, if "efficiency" is to be used as a criterion which is normally higher in a better option, in the IDMI calculation, "1/efficiency" is more appropriate. 


\begin{tabular}{|l|c|c|c|c|}
\hline & Option 1 & Option 2 & Option 3 & Option 4 \\
\hline Capital costs & 1 & 1.2 & 0.9 & 1.4 \\
\hline $\mathrm{CO}_{2}$ emission & 1 & 0.9 & 1.1 & 0.8 \\
\hline 1/(Plant efficiency) & 1 & 1 & 1.1 & 0.9 \\
\hline OHS risks & 1 & 0.95 & 1 & 1 \\
\hline 1/(sustainability) & 1 & 1 & 1 & 0.9 \\
\hline Others losses/risks & 1 & 1 & 1 & 0.9 \\
\hline Total Value & $\mathbf{6}$ & $\mathbf{6 . 0 5}$ & $\mathbf{6 . 1}$ & $\mathbf{5 . 9}$ \\
\hline
\end{tabular}

Table 1: A hypothetical example

In this case, the value of each criterion among the options is not the help of IDMI. With the information in Table 1, IDMIs can be much different. The selection is relatively hard to make without calculated and will depend on which criteria are "critical"
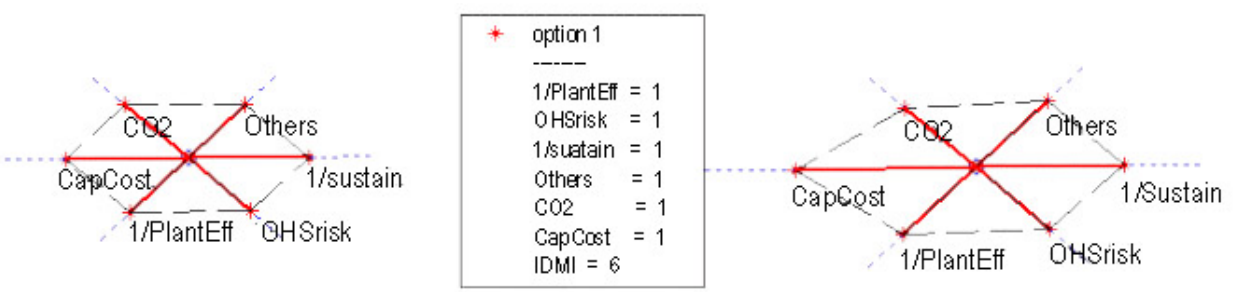

$\begin{array}{|ll|}* \text { option } 2 & \\ ---- & \\ \text { 1/PlantEff } & =1 \\ \text { OHSrisk } & =0.95 \\ 1 / \text { Sustain } & =1 \\ \text { Others } & =1 \\ \text { Co2 } & =0.9 \\ \text { CapCost } & =1.2 \\ \text { IDMI }=6.05 & \end{array}$
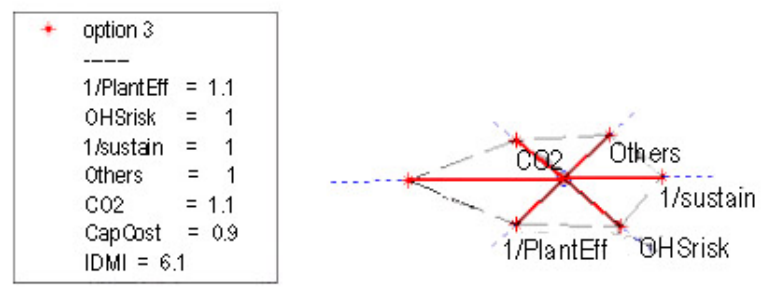

\begin{tabular}{|ll|}
\hline+ option 4 & \\
---- & \\
1/PlantEf & $=0.9$ \\
OHSrisk & $=1$ \\
1/sustain & $=0.9$ \\
Others & $=0.9$ \\
CO2 & $=0.8$ \\
CapCost & $=1.4$ \\
IDMI $=5.9$
\end{tabular}

Figure 3: IDMI results without CC selected. 


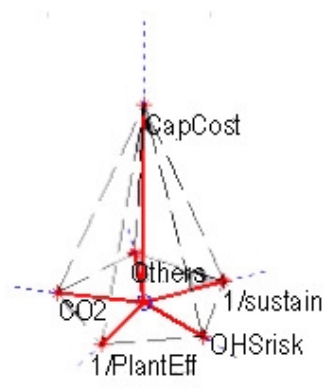

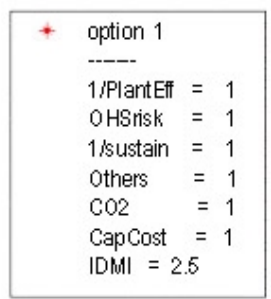

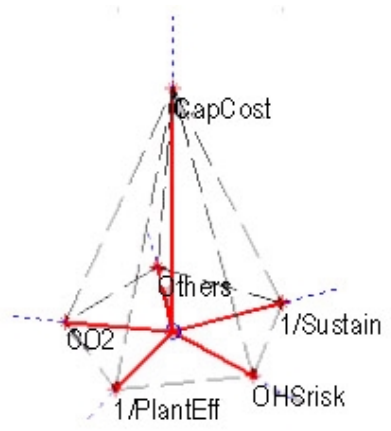

$\begin{array}{|ll|}* \text { option } 2 & \\ -1 \text { /PlantEf } & =1 \\ \text { OHSrisk } & =0.95 \\ \text { 1/sustain } & =1 \\ \text { Others } & =1 \\ \text { CO2 } & =0.9 \\ \text { CapCost } & =1.2 \\ \text { IDMI }=2.91\end{array}$
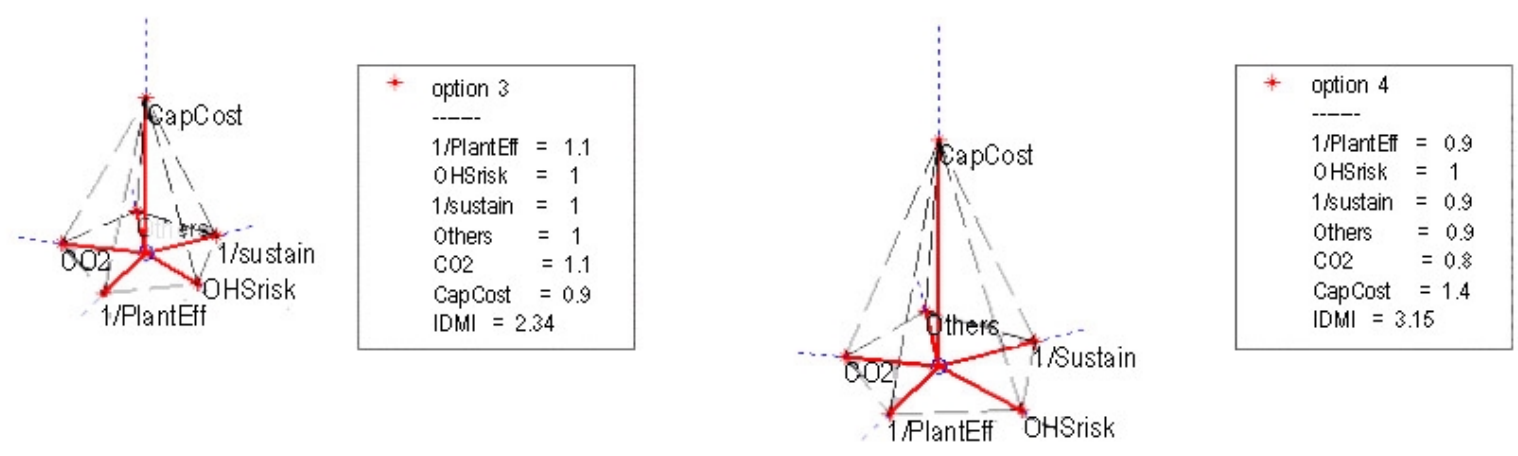

Figure 4: IDMI results with "capital cost" as the CC in the decision making process.

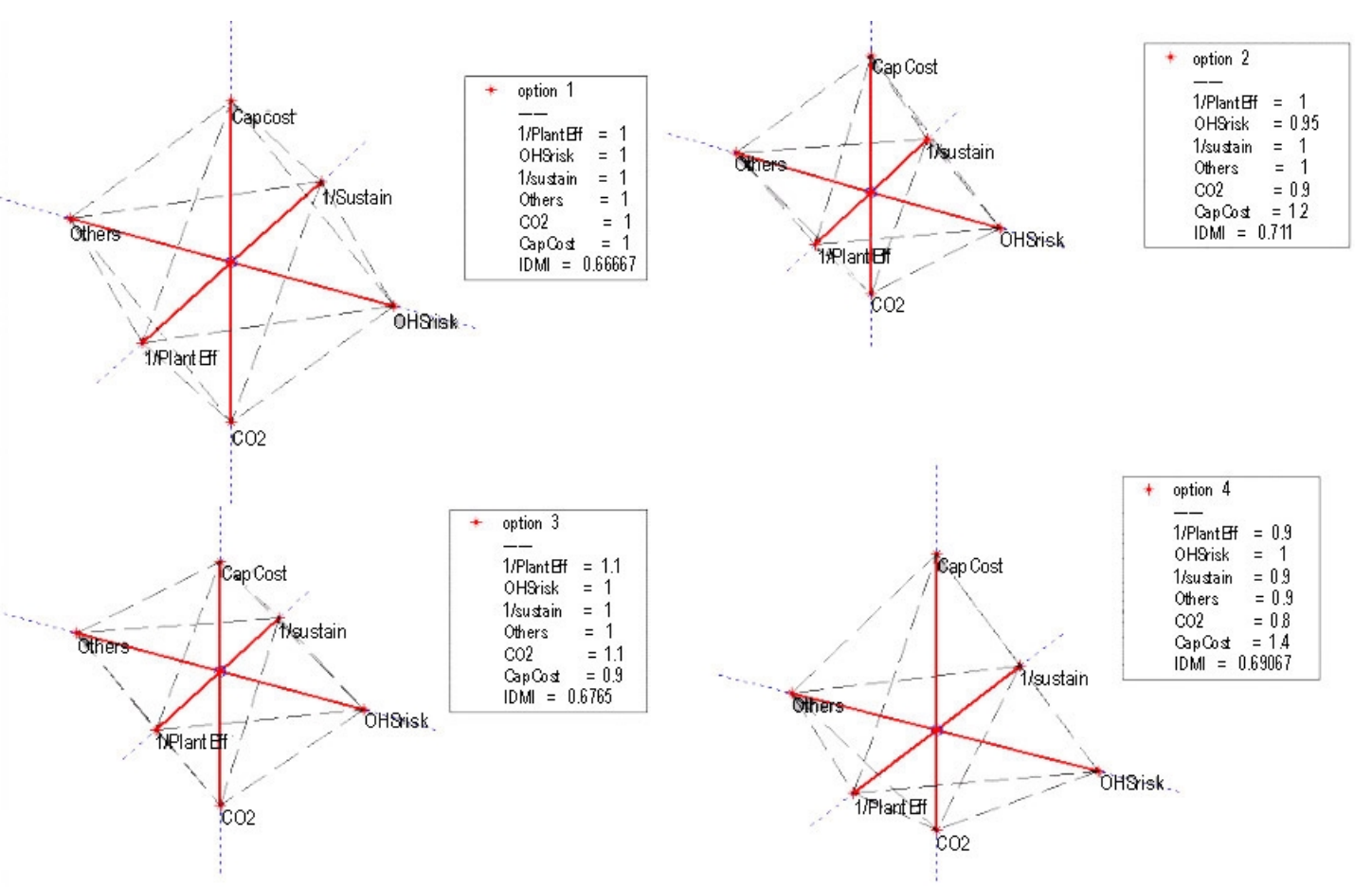

Figure.5: IDMI results using "capital cost" and " $\mathrm{CO}_{2}$ emission" as two CCs. 
If all criteria are considered of equal importance, i.e. no CC selected, then Fig. 3 gives the IDMI results. In this case, option 4 appears the best choice. Although in this case the IDMI produces the same ranking results as the simple sum of the all criteria values (i.e. the last row of Table 1), the IDMI results have a better visual insight than the table, especially when printing the IDMI illustration in one overlaid diagram.

If the "capital cost" is the major concern, and was selected as the $\mathrm{CC}$, the choice may be option 3, as shown in Fig.4. If "capital cost" and " $\mathrm{CO}_{2}$ emission" were of primary concern, then IDMI using 2 CC would help (see Fig. 5). The choice now appears to be option1. Comparing the various options with different CCs will make the ranking change to reflect priorities.

\section{Discussion}

It is a valid question to ask what makes the proposed IDMI method superior to a conventional tabular method?

Comparing the IDMI concept with the traditional tabular method using conventional weightings, sometimes the same ranking will result. But in many cases, they will differ. The ranking results of the tabular method are highly dependant on the weighting factors given to the $\mathrm{CC}$, and this weighing factor makes the process highly subjective. The IDMI eliminates this human interfering process and is hence more objective and nonpolitical.

In the simple weighing concept, the total value is the sum of values of all criteria with a weighting factor multiplied to the critical criteria:

$$
T . V .=\left(\sum_{i=1}^{n-1} C_{i}\right)+w C_{n}
$$

and where two critical criteria are selected, then:

$$
T . V .=\left(\sum_{i=1}^{n-2} C_{i}\right)+w_{1} C_{n-1}+w_{2} C_{n}
$$

where:

T.V. = total value;

$\mathrm{n}=$ the number of the criteria;

$\mathrm{w}=$ the weighting factor;

$C_{i}=$ the value of the normal criteria

$\mathrm{C}_{\mathrm{n}}=$ the first $\mathrm{CC}$;

$C_{n-1}^{n}=$ the second $C C$;

Compared with equations 2 and 3 , the (mathematical) relationship between the $\mathrm{CC}$ and normal criteria are different in the IDMI concept to that used in conventional weightings (equations 4 and 5). It can be seen that the weightings of selected CC have been interlinked with all other normal criteria through the IDMI geometry structure. The next example shows clearly the difference between them.
In the case shown in Table 2, "capital cost" is selected as the only critical criteria (CC) and the remaining five are normal criteria. In option 3, the critical criteria (capital cost) is doubled; in option 4, the non-critical criteria (other risks) is doubled, both compared to the reference option 2 . The values of all other criteria remain the same.

Selection is to be made between options 3 and 4 . If using a tabular method with simple weightings (i.e. using a weighting factor of 2 to capital cost), the result recommends the option 3 because it has the lower total value. But it is obviously misleading, and does not appropriately reflect the criticality of the CC. If using the IDMI method, the recommended choice is option 4 (see Fig. 6). This implies that we prefer the worse normal criteria option rather than the worse CC option. This shows that the criticality of the $\mathrm{CC}$ is prioritized as a direct result of the IDMI approach. Moreover, the results from the tabular method depend on the magnitude of weighting factor given. If for instance, the weighting factor 20 instead of 2 was used, the results would be different. 


\begin{tabular}{|l|c|c|c|c|}
\hline & $\begin{array}{c}\text { Option1: } \\
\text { Non-existing } \\
\text { ideal case }\end{array}$ & $\begin{array}{c}\text { Option 2: the } \\
\text { reference }\end{array}$ & $\begin{array}{c}\text { Option 3: } \\
\text { double the } \\
\text { capital costs" }\end{array}$ & $\begin{array}{c}\text { Option 4: double } \\
\text { the "Other } \\
\text { losses/risks" }\end{array}$ \\
\hline $\begin{array}{l}\text { Capital costs } \\
\text { (critical criterion) }\end{array}$ & 1 & 0.5 & 1 & 0.5 \\
\hline $\mathrm{CO}_{2}$ emission & 1 & 4 & 4 & 4 \\
\hline 1/(Plant efficiency) & 1 & 2 & 2 & 2 \\
\hline OHS risks & 1 & 1 & 1 & 1 \\
\hline 1/(sustainability) & 1 & 5 & 5 & 5 \\
\hline Others losses/risks & 1 & 4 & 4 & 8 \\
\hline $\begin{array}{l}\text { Total with CC given } \\
\text { weighting factor by 2 }\end{array}$ & $\mathbf{7}$ & $\mathbf{1 7}$ & $\mathbf{1 8}$ & $\mathbf{2 1}$ \\
\hline
\end{tabular}

Table 2: Another hypothetical example
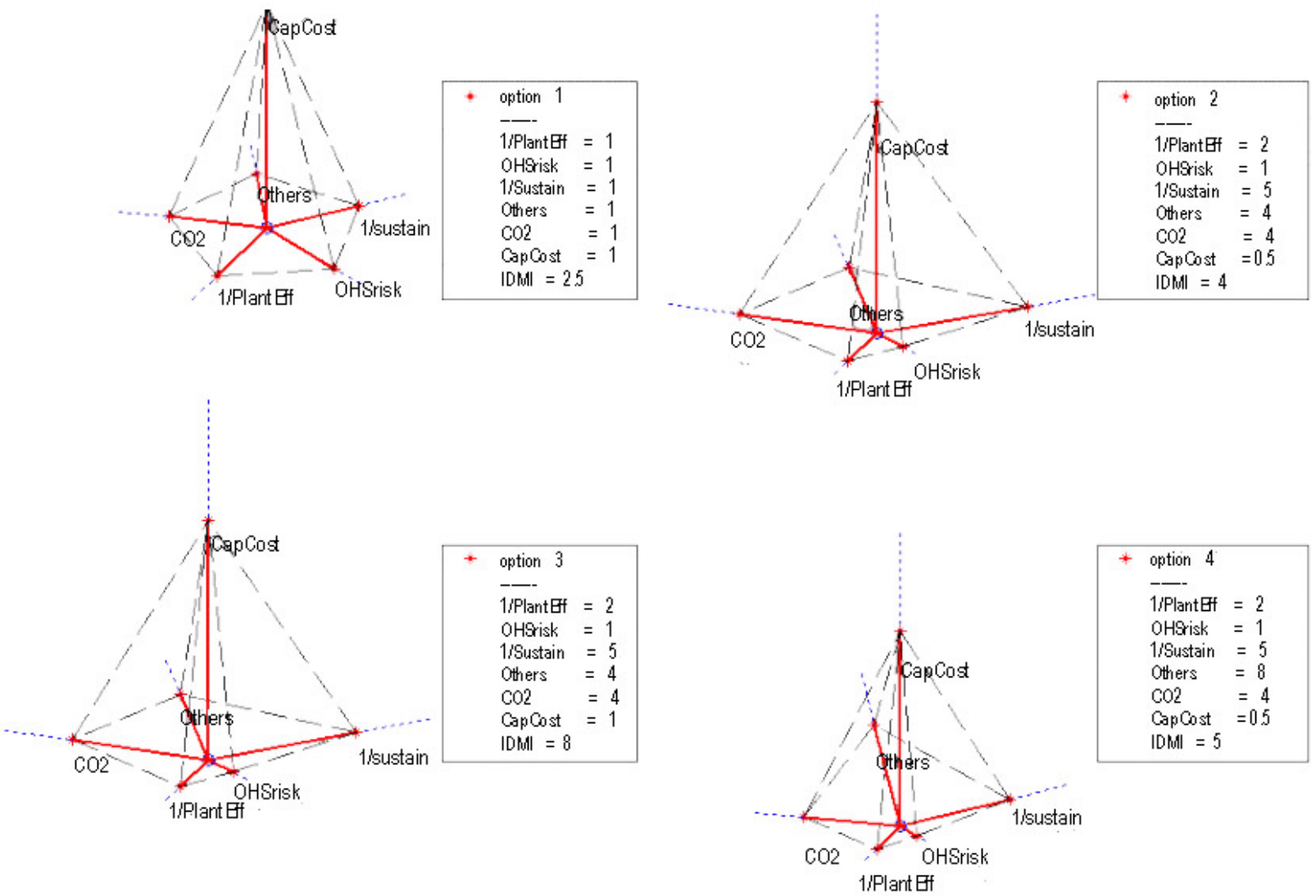

Figure 6: IDMI results for the second example.

The IDMI concept is not attempting to make the decision automatically, but provides a scale to measure the various options that are available. IDMI values that ate close indicate the choice is even. By looking at a number options the range of IDMI values (and hence the significance of the comparison) will be clear. 
The authors put forward the IDMI concept for post assessment data conversion purposes. It is more user friendly, more effective and powerful to reveal the relationship among the criteria than traditional tabular or bar chart methodologies. The definition/design of the IDMI guarantees the criticality of the CC in the calculation of IDMI. The proposed IDMI concept with the illustration is a simple and transparent way to assist the decision maker to compare multi-criteria options. It interlinks the information from multidisciplinary assessments and reveals visually the characteristics of every option in each selection criteria. Most importantly, the IDMI concept avoids the subjective weighting process which is the most difficult part of decision making after the $\mathrm{CC}$ has been selected. In the IDMI concept, once the $\mathrm{CC}(\mathrm{s})$ is chosen, weighting is automatically given and the priority consideration will be guaranteed for these criteria.

Therefore the use of IDMI forms a strategic decision making tool that subsumes techniques such as CBA, LCA and EIA and enables multiple criteria to be evaluated in an objective and dispassionate manner. Problems of manipulation and bias are largely avoided.

\section{REFERENCES}

Abelson, P. W. (1996), Project Appraisal and Valuation of the Environment: General Principles and Six Case-Studies in Developing Countries, MacMillan.

Chen, M. J. and Huang, G. H. (2001): A derivative algorithm for inexact quadratic program- application to environmental decision making under uncertainty. European Journal of Operational Research, Vol. 128, Issue 3, pp570-586.

Ding, G. and Langston, C. (2002), A Methodology for Assessing the Sustainability of Construction Projects and Facilities. ICEC 3rd World Conference, Melbourne.

Hanley, N. (1992), Are There Environmental Limits to Cost Benefit Analysis? Environmental and Resource Economics, vol. 2, pp.33-59.

Khan, F. I. et al: Green Pro-I (2002): A Risk Based Life Cycle Assessment and Decision Making Methodology for Process Plant Design, Environment Modelling and Software, in press.

Nijkamp, P. , Rietveld, P. and Voogd, H. (1990), Multicriteria Evaluation in Physical Planning, North-Holland.

Van Pelt, M. J. F. (1993), Ecological Sustainability and Project Appraisal, Averbury.

Vignes, R. P. (1999): Limited Life Cycle Analysis: A Tool for the Environment Decision Making Toolbox, Strategic Environment Management, Vol. 1 Issue 4, pp297-332.

Weidema, B. P. (2000): Environmental Assessment of ProductsA textbook on life cycle assessment. The Finnish Association of Graduate Engineers TEK. 